\title{
Screening and Evaluation Tool (SET) Users Guide
}

\author{
Layne Pincock
}

October 2014

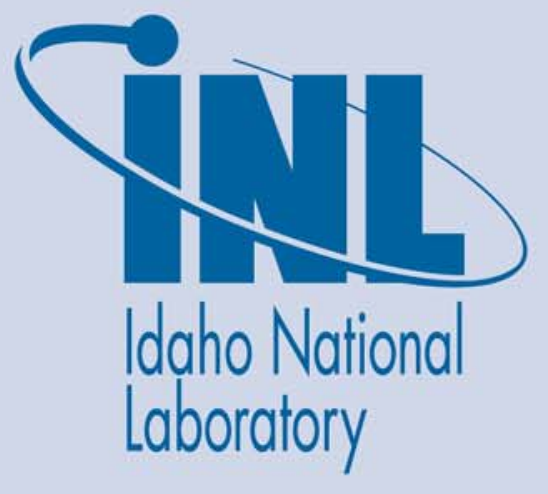

The INL is a U.S. Department of Energy National Laboratory operated by Battelle Energy Alliance 
INL/EXT-14-32118

\title{
Screening and Evaluation Tool (SET) Users Guide
}

\author{
Layne Pincock
}

October 2014

\section{Idaho National Laboratory \\ Idaho Falls, Idaho 83415}

http://www.inl.gov

Prepared for the

U.S. Department of Energy

Office of Nuclear Energy

Under DOE Idaho Operations Office

Contract DE-AC07-05ID14517 


\section{Screening and}

Evaluation Tool (SET)

Users Guide

Fuel Cycle Research \& Development

Prepared for

U.S. Department of Energy Fuel Cycle Options Campaign Layne Pincock

Idaho National Laboratory October 29, 2014 FCRD-FCO-2014-00395 
DISCLAIMER

This information was prepared as an account of work sponsored by an agency of the U.S. Government. Neither the U.S. Government nor any agency thereof, nor any of their employees, makes any warranty, expressed or implied, or assumes any legal liability or responsibility for the accuracy, completeness, or usefulness, of any information, apparatus, product, or process disclosed, or represents that its use would not infringe privately owned rights. References herein to any specific commercial product, process, or service by trade name, trade mark, manufacturer, or otherwise, does not necessarily constitute or imply its endorsement, recommendation, or favoring by the U.S. Government or any agency thereof. The views and opinions of authors expressed herein do not necessarily state or reflect those of the U.S. Government or any agency thereof. 


\section{SUMMARY}

This document provides a description of the use of the Screening and Evaluation Tool (SET) which was used for the Nuclear Fuel Cycle Evaluation and Screening - Final Report (October 8, 2014). The SET tool is prepopulated with data from this report. It is able to reproduce many of the results presented in the Evaluation and Screening report. The SET tool is one of several tools developed to support the Evaluation and Screening Study and is provided to the user for the main purpose of facilitating exploration of the sensitivity of the results to changes in perspective about the relative importance of benefit criteria and metrics. The user can also add new fuel cycle options with their corresponding metric data which can then be compared with the Evaluation Groups used in the Study. 


\section{CONTENTS}

SUMMARY iii

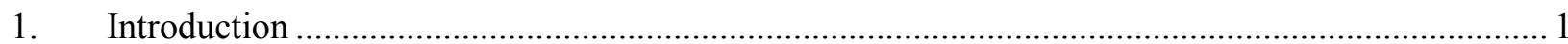

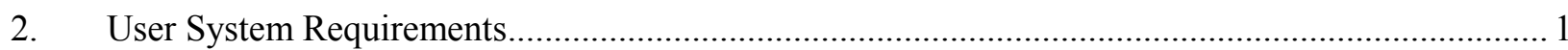

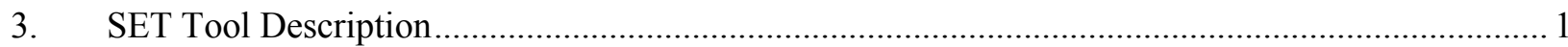

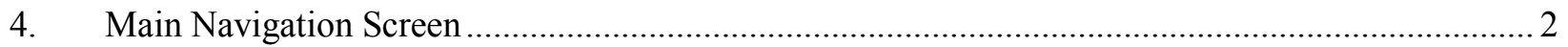

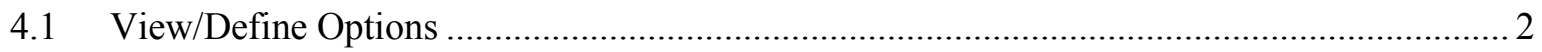

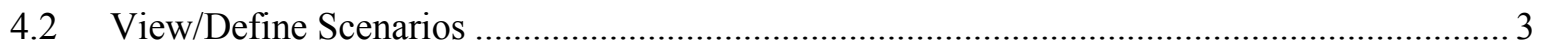

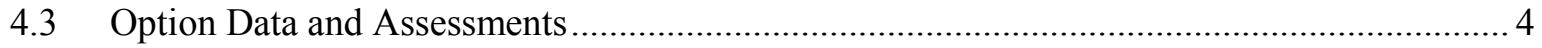

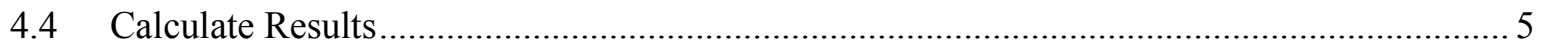

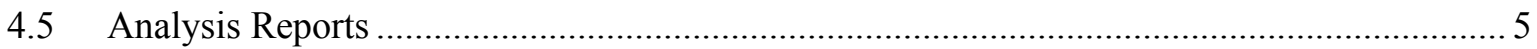

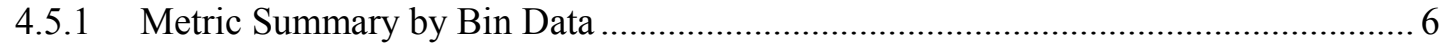

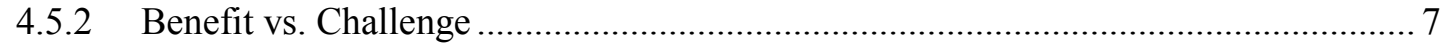

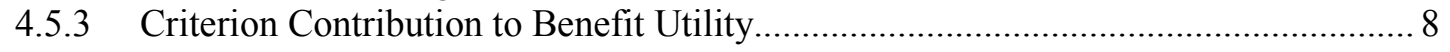

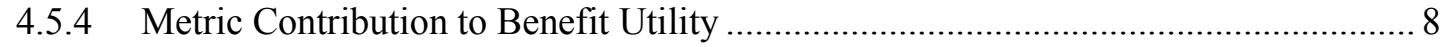

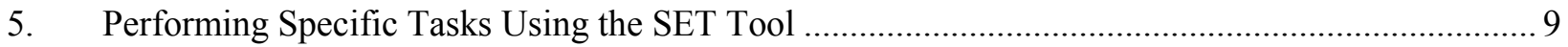

5.1 Determining Which Evaluation Group Best Represents My Fuel Cycle............................... 9

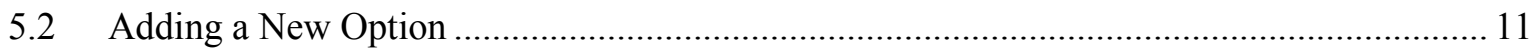

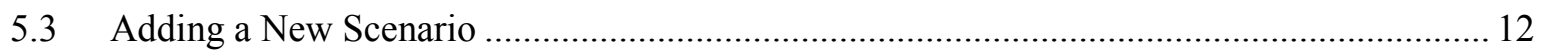

\section{FIGURES}

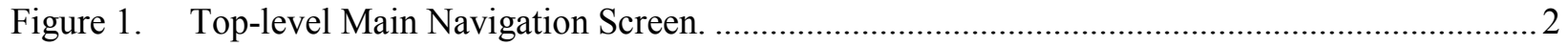

Figure 2. View/Define Options Screen..................................................................................... 3

Figure 3. View/Define Scenarios Screen..................................................................................... 4

Figure 4. Option Data and Assessments Screen. ........................................................................ 5

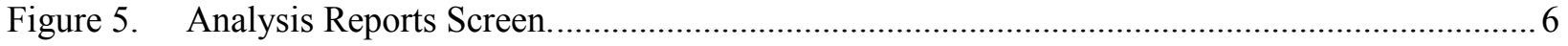

Figure 6. Metric Summary by Bin Data Report Example. …......................................................... 6

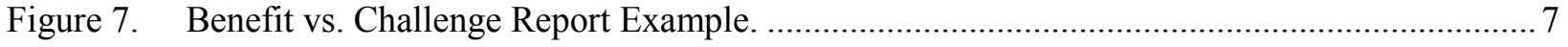

Figure 8. Criterion Contribution to Benefit Utility Report Example................................................... 8

Figure 9. Metric Contribution to Benefit Utility Report Example ........................................................ 9

Figure 10. First Step in "Find Which Evaluation Group My Fuel Cycle Is In" Process. ........................ 10

Figure 11. Final Screen for Identifying Which Evaluation Group Your Fuel Cycle Is In...................... 11

Figure 12. Adding Metric Values for a New Option. ........................................................................ 12 


\section{SCREENING AND EVALUATION TOOL (SET) USER'S GUIDE}

\section{Introduction}

This document provides a description of the use of the Screening and Evaluation Tool (SET) which was used for the Nuclear Fuel Cycle Evaluation and Screening - Final Report (October 8, 2014). The SET tool is prepopulated with data from this report. It is able to reproduce many of the results presented in the Evaluation and Screening report. The SET tool is one of several tools developed to support the Evaluation and Screening Study and is provided to the user for the main purpose of facilitating exploration of the sensitivity of the results to changes in perspective about the relative importance of benefit criteria and metrics. The user can also add new fuel cycle options with their corresponding metric data which can then be compared with the Evaluation Groups used in the Study.

The SET software is a tool for comparing multiple fuel cycle options against a common set of criteria and metrics. It does this using standard multi-attribute utility decision analysis methods. See Section A-3 of Appendix A in the Evaluation and Screening report for further detail on the equations and functions used in SET. SET includes the ability to generate various output reports to analyze results as well as a module to help the user determine which Evaluation Group best matches the user's fuel cycle of choice.

The SET software was developed in an iterative fashion by first obtaining an initial set of requirements from members of the Evaluation and Screening Team (EST) and developing the initial SET software. Several demonstrations occurred with EST members on different occasions during which the requirements were further developed and solidified.

The document is organized by first going through the main steps or flow of the tool and describing the screens that are used (Section 3). Then specific tasks (such as how to add a new option) are described in Section 4.

\section{User System Requirements}

SET was developed in Microsoft Excel 2010. The tool itself is an Excel spreadsheet with macros. Therefore, to use SET the user will need to have Microsoft Excel 2007 or newer installed in Windows XP or newer and enable macros (macros must be enabled for SET to work).

SET will work on the Mac version of Microsoft Excel 2011 in MacOS 10.7 or newer operating system, but it was not fully tested under this environment so no guarantees are made as to its functionality.

The recommended operating environment is Excel 2010 under Windows 7 (as this is the platform in which it was developed and tested.

\section{SET Tool Description}

The SET tool was built as a general purpose tool that supports the evaluation of a group of options against a set of criteria and metrics. The evaluation metrics are items used to measure the performance of an option. The evaluation metrics roll up into higher level evaluation criteria as described in the Evaluation and Screening report (Appendix F, Section F-1). SET takes the list of options and their corresponding metric performance data and uses a shape function to determine a utility value for each metric. These metrics are combined using trade-off factors defined by the scenario. These calculated combined utility 
values can then be use to compare one option to another. The SET tool is designed to step the user through the process of evaluation of fuel cycle options.

\section{Main Navigation Screen}

The purpose of this main screen is to provide a central navigation point for the tool. All parts of the tool can be accessed from this main screen. Each sub-screen has a navigation arrow to go "Back to the Main Menu." This screen also provides overview information for the user.

It is recommended that you keep the original file unchanged (so you can always start over). Each time you want to make changes to the SET tool save the Excel file as a new file and continue from there. (It is also recommended that the user save all output report files into the same folder as your new copy of SET, thus keeping the input file together with all the output files.) The top level screen shown in Figure 1 is the starting point for using SET.

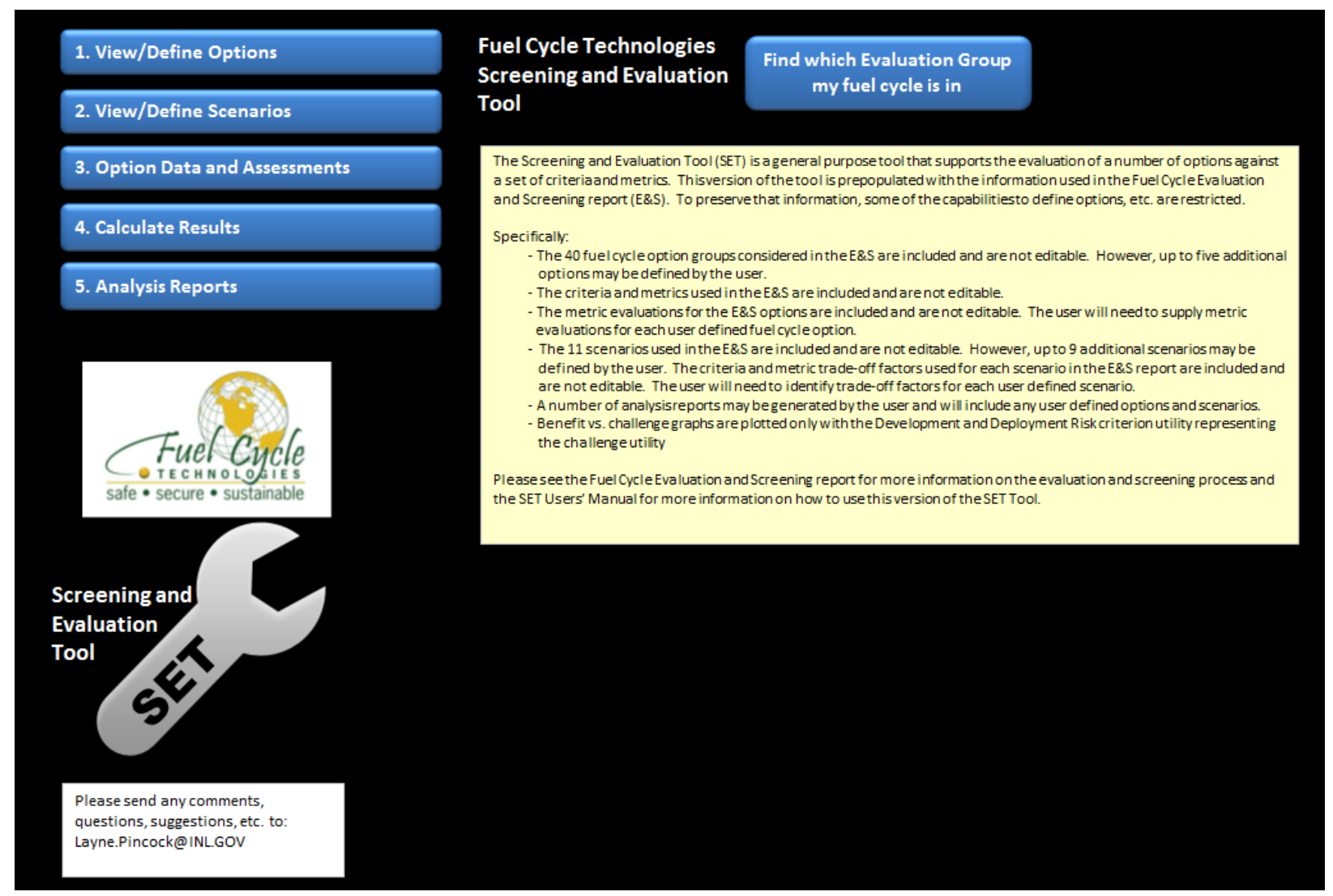

Figure 1. Top-level Main Navigation Screen.

\subsection{View/Define Options}

The purpose of this screen is to view the list of existing options that were developed and used in the Evaluation and Screening report and to allow the user to enter his/her own options (up to five new options). By clicking on the 1. View/Define Options button, the user is taken to the view/define options page. This page is simply a list of options to be evaluated by the tool. The Evaluation Group column is required and the other columns are optional for describing the options. 
The tool is prepopulated with the 40 Evaluation Groups from the fuel cycle study performed by the Evaluation and Screening Team. The first option is the "Basis of Comparison", representing the current U.S. fuel cycle as it would be completely implemented including disposal of the spent nuclear fuel.

If you would like to add an option simply type in the new option in row 41. In the Evaluation Group column enter each new option sequentially (e.g. EG41, EG42, etc.). You may add additional options up to 45 total options. Fill in the options sequentially and do not leave any blank rows. (See Section 5.2 for completing all the steps needed for adding a new option.) The green highlighted areas show the user which cells are changeable and not locked down.

Make sure to check the checkbox for any new option if you want it included in the analysis. If you would like to exclude any of the options from the analysis, you can uncheck the checkbox in the column 'Include in evaluation?'. This is useful for focusing on a subset of the options.

When you are done reviewing or making changes in this screen, press the Back to main menu button to return to the main menu or the Next button to skip directly to the screen for the next step of using the SET tool.

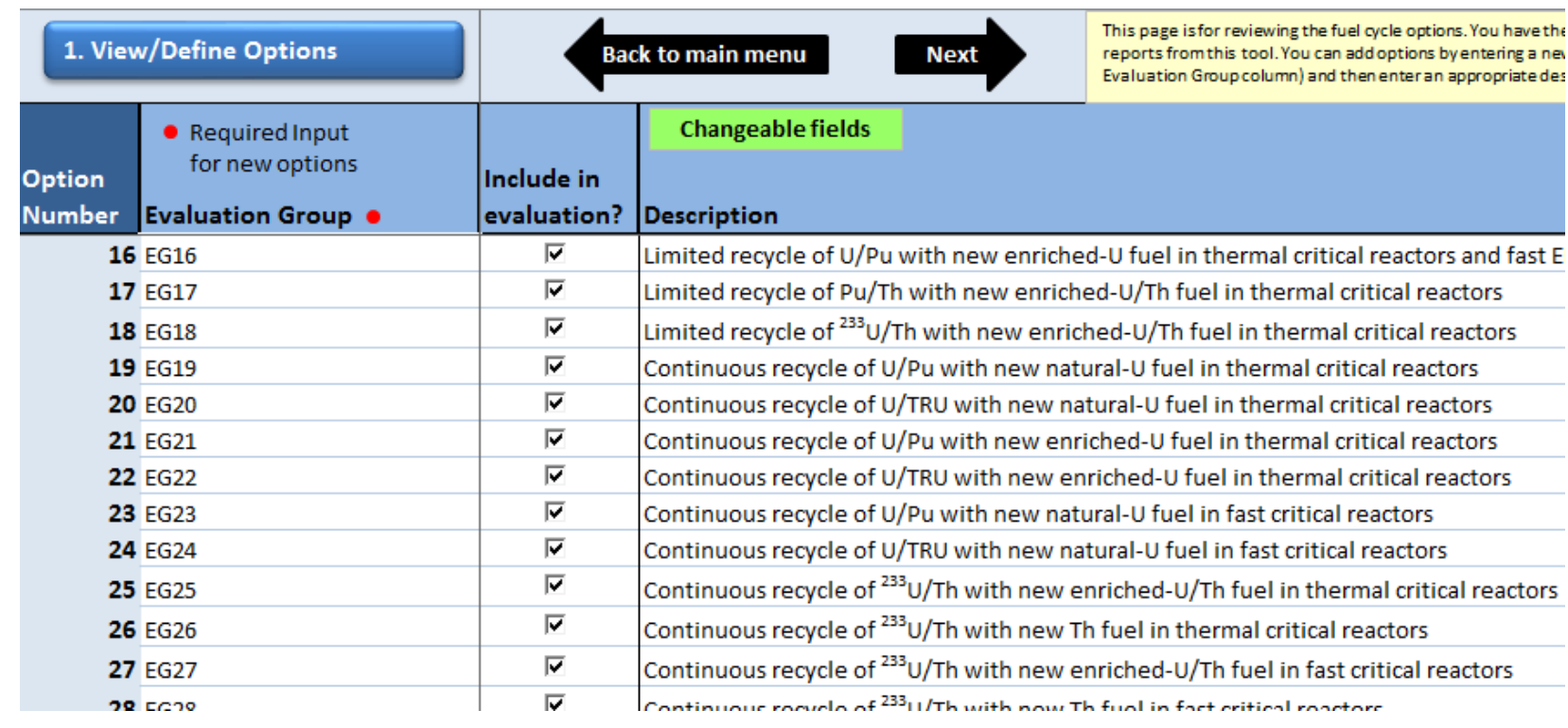

Figure 2. View/Define Options Screen.

\subsection{View/Define Scenarios}

By pressing the 2. View/Define Scenarios button, SET will take you to the screen shown below in Figure 3. The purpose of this page is to view the existing predefined scenarios used in the Evaluation and Screening report and to allow the user to define his/her own scenarios. To start off with, this page shows the list of criteria and metrics used in the evaluation process and how they relate to each other. On the far left of this page you can see the criteria followed by the metrics. The row spacing and metric index indicate which metrics are included with each criterion. The "challenge" criteria are listed at the top and the "benefit" criteria are listed at the bottom. A detailed discussion of the criteria and metrics is found in Appendices D and E, and in Section F-1 of Appendix F of the Evaluation and Screening report.

The trade-off factors defined in this step determine how the metrics for each of the options are combined at the criteria level and then the criteria results combined into a composite benefit utility for comparison between the Evaluation Groups. 


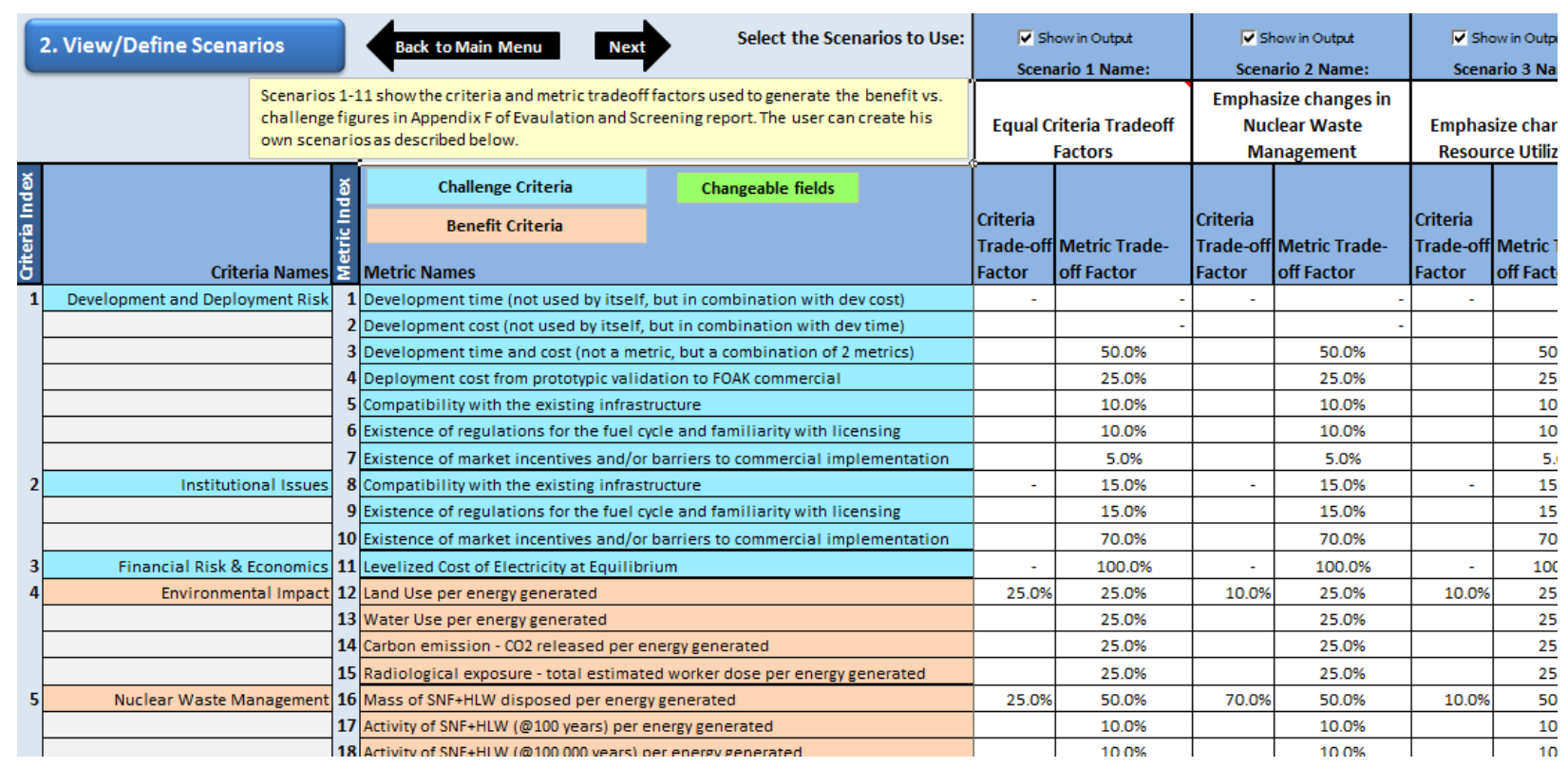

Figure 3. View/Define Scenarios Screen.

The first scenario (just to the right of the metrics) is the even weight scenario. The criteria and metric trade-off factors for the 11 scenarios used in the Evaluation and Screening report cannot be changed. There is a check box at the top of the screen that allows you to select which scenarios you want to include in your analysis. You can have up to 20 scenarios total. New scenarios are entered toward the right side of the screen. If you add a new scenario, add it in the first blank column to the right side of the screen. The fields you are allowed to change are highlighted in green. Enter the name of each new scenario at the top of the screen and then enter the desired trade-off factors for each criterion and enter the metric tradeoff factors. All of the criteria trade-off factors should add to $100 \%$, and the metric trade-off factors for each criterion should also add to $100 \%$. There are some checksum values calculated at the bottom of the screen to give feedback on if your trade-off factors sum to $100 \%$.

When you are done reviewing or making changes in this screen, press the Back to main menu button to return to the main menu or the Next button to skip directly to the screen for the next step of using the SET tool.

\subsection{Option Data and Assessments}

The purpose of this screen, shown in Figure 4, is to enter the metric data for any new option defined in Step 1 and to review the data results for each of the predefined Evaluation Group options and their associated metrics and how the shape functions convert each data result into a utility value. Each of the metrics was divided into bins. These bins each have a range which together span the range of possible results for all the fuel cycle options. In the upper left portion of the screen you can navigate through each of the options and metrics using the arrow buttons. You can quickly jump to a specific option by clicking on the option number and entering the option number you would like to jump to. The plot in the middle of the screen shows the shape function and how the data value maps to a utility value (dotted lines). Metric data bin assignments are locked for the 40 predefined Evaluation Groups. Only the bin assignments of new options can be entered or changed here. See Section 5.2 for detailed instructions on how to make bin assignments for a new option. 
When you are done reviewing or making changes in this screen, press the Back to main menu button to return to the main menu or the Next button to skip directly to the screen for the next step of using the SET tool.

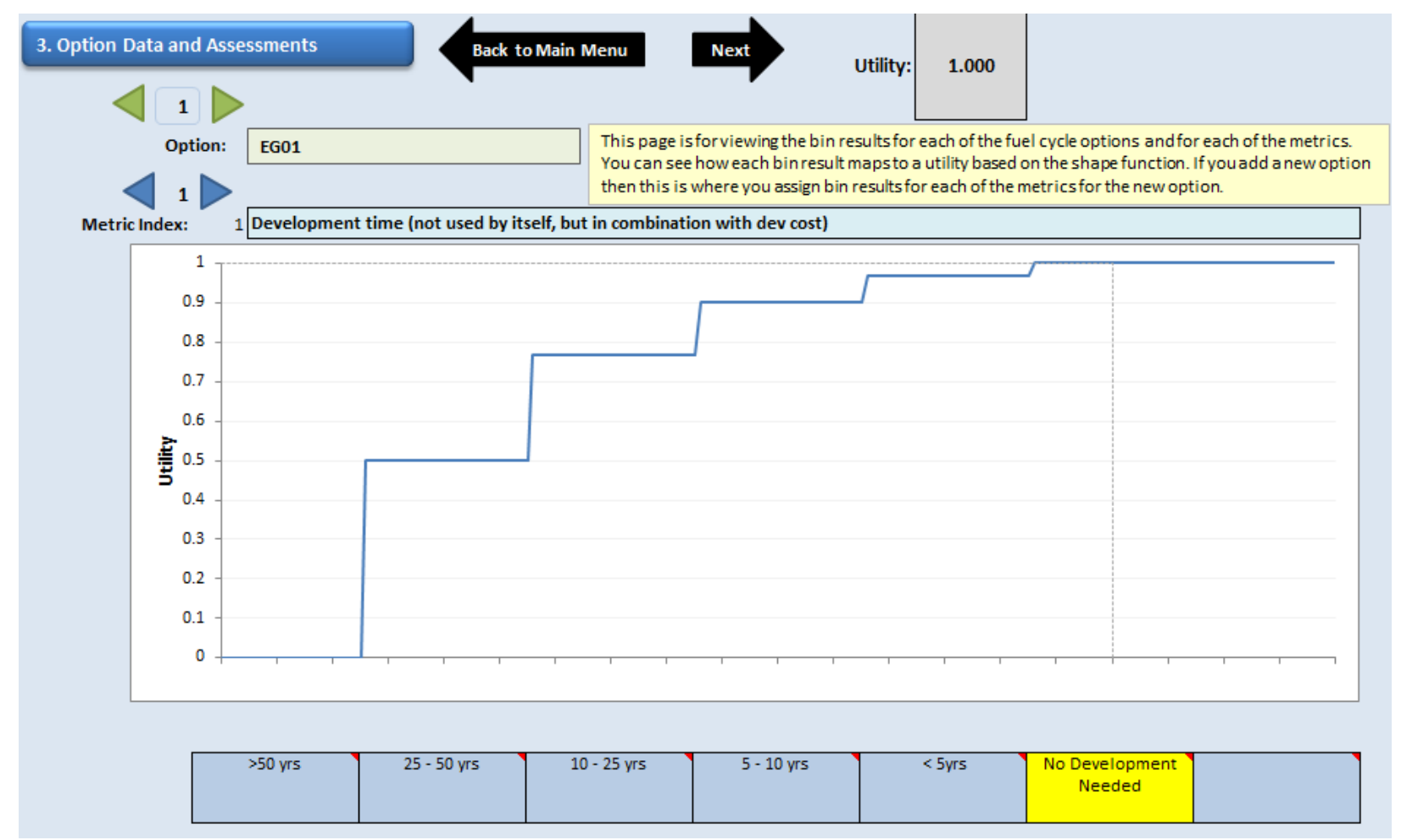

Figure 4. Option Data and Assessments Screen.

\subsection{Calculate Results}

This button calculates the results of the evaluation including any entries for new options, scenarios, and new option metric data that were made in the previous steps. It may take a few minutes to do all the updates and calculations depending the speed of your computer. You can see the progress in the lower left of your screen. When it is complete, the SET tool will take you to the final Analysis Reports screen.

\subsection{Analysis Reports}

After calculating results, the SET tool will take you to the Analysis Reports screen as shown below. The purpose of this screen is to run various reports of your choice and analyze the results. In this screen there are various output reports to choose from in the menu. Choose the desired report in the list and press the Run button to generate the report. Some reports require a lot of calculation and may take a minute or two. Each of the reports is discussed in the subsequent sections below. 


\section{Analysis Reports}

\begin{tabular}{|l|l|}
\hline Select Report from pick list and click Run to generate report & $\begin{array}{l}\text { This report displays a bar graph of all options for each } \\
\text { metric by the bin result. }\end{array}$ \\
\hline Metric Summary by bin data
\end{tabular}

It is recommended as good practice that as you do an analysis, that you save the main SET file in a folder (using "Save as") and then save all output report files in the same folder to keep them together with the main file thus keeping the inputs with the outputs.

Figure 5. Analysis Reports Screen.

\subsubsection{Metric Summary by Bin Data}

The first report in the menu is the Metric Summary by bin data report. This report shows the summary of the bin data for each of the metrics and for each of the options selected. The bin data is shown on the $y$-axis of each of the graphs. There is a sheet for each of the metrics showing each option's data value (by bin). This report generates a new Excel spreadsheet with a sheet for each of the metrics. This report shows the bin data for each option based on the bin structure for each metric as defined in Appendix D of the Evaluation and Screening report.

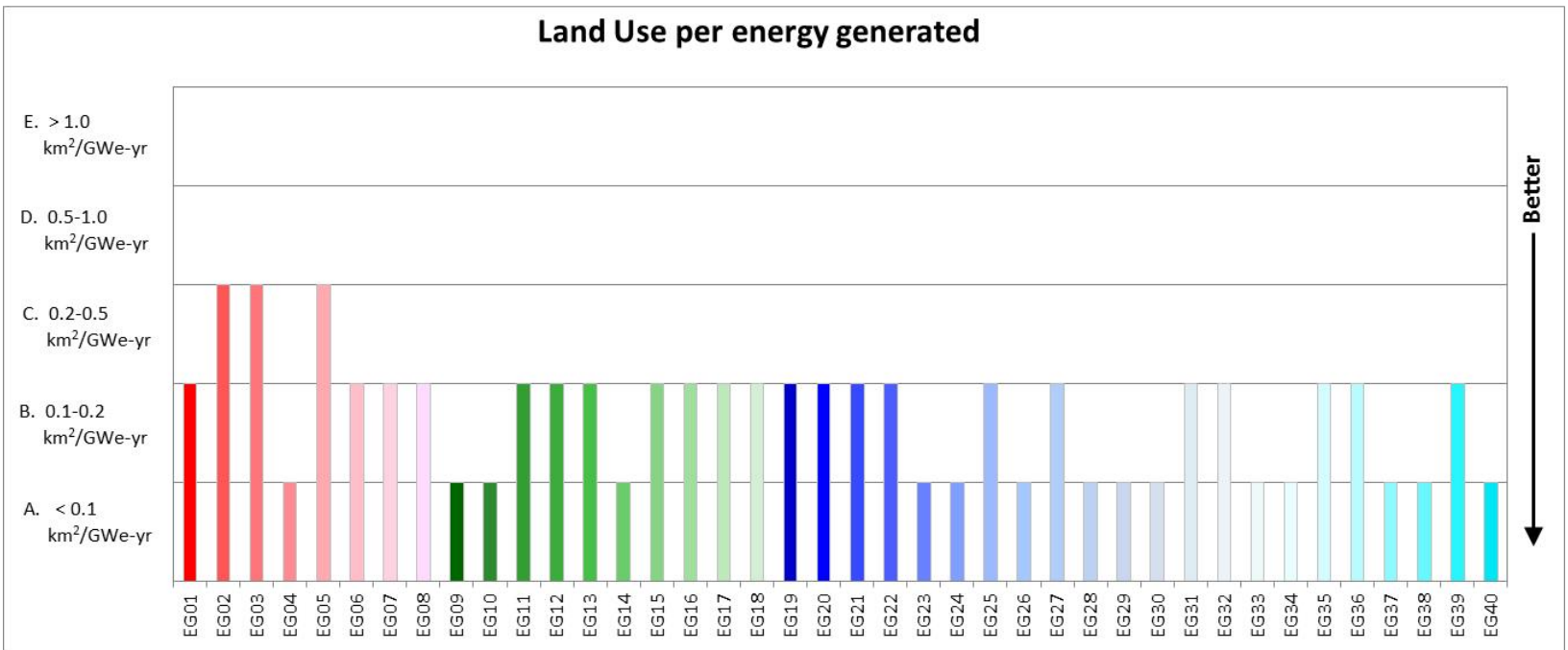

Figure 6. $\quad$ Metric Summary by Bin Data Report Example. 


\subsubsection{Benefit vs. Challenge}

The second report in the menu is the Benefit vs. Challenge report that plots benefit utility vs. challenge utility as discussed in the Evaluation and Screening report and Appendices E and F. This report generates a new Excel spreadsheet file with a separate chart for each scenario defined in 2. View/Define Scenarios, along with a Data tab containing a listing of all the plotted data for each scenario. This report generates a plot of the selected benefit criteria against the challenge criterion of Development and Deployment Risk. When you run this report, SET will ask you which challenge criteria you want for the x-axis. Criterion 1 for Development and Deployment Risk is the only choice, so simply select and click OK to run the report. When the calculations are finished, a graph similar to Figure 7 will appear on your screen. Each plotted point is an option (or Evaluation Group) and is labeled (some labels overlay one another and can be moved to separate them on the plot by clicking on the label and dragging it to the desired location). The large arrows on the graph help the user interpret the results. The orange/yellow lines in Figure 7 illustrates example thresholds: all Evaluation Groups above this line would be identified as promising groups for a decision-maker who determines that the line represents sufficient improvement. For some scenarios, no Evaluation Groups may be identified as potentially promising based on the example threshold(s), just as for some Criteria, no promising options were identified in the Evaluation and Screening report, as discussed in the report and Appendices A and F. The Basis of Comparison (EG01) is colored red for ease of identification.

To examine the results for a single benefit criterion at a time using this report, just define a scenario where the desired criterion has $100 \%$ of the criteria trade-off value and name the scenario appropriately.

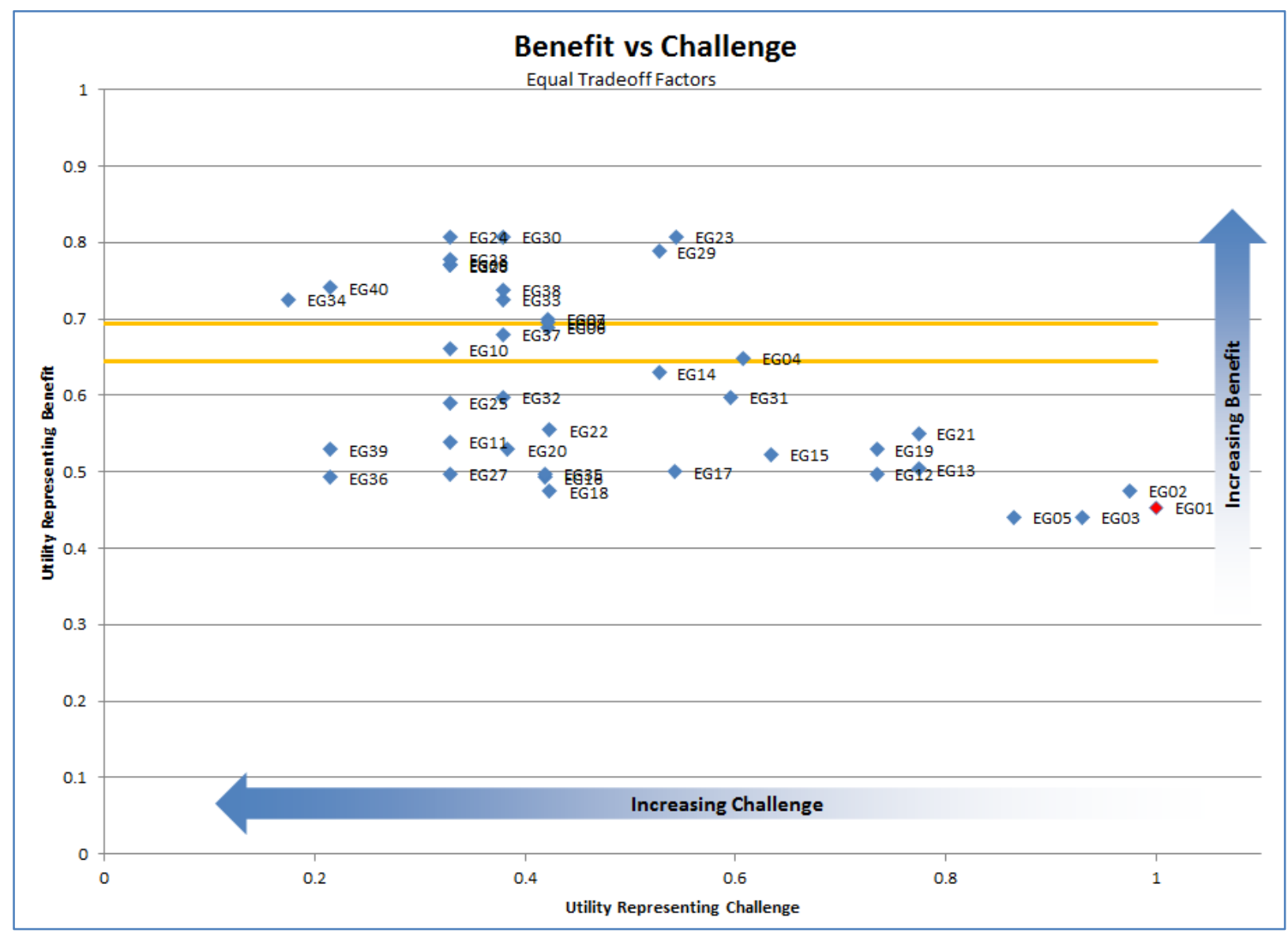

Figure 7. Benefit vs. Challenge Report Example. 


\subsubsection{Criterion Contribution to Benefit Utility}

The third report in the list is the Criterion Contribution to Benefit Utility report. This report generates a new spreadsheet with one summary column graph of each of the options sorted in order of decreasing benefit utility for each scenario. Within each column, the colors show the contribution of each of the criteria. This report generates a worksheet and graph for each of the scenarios selected in step 2. View/Define Scenarios.

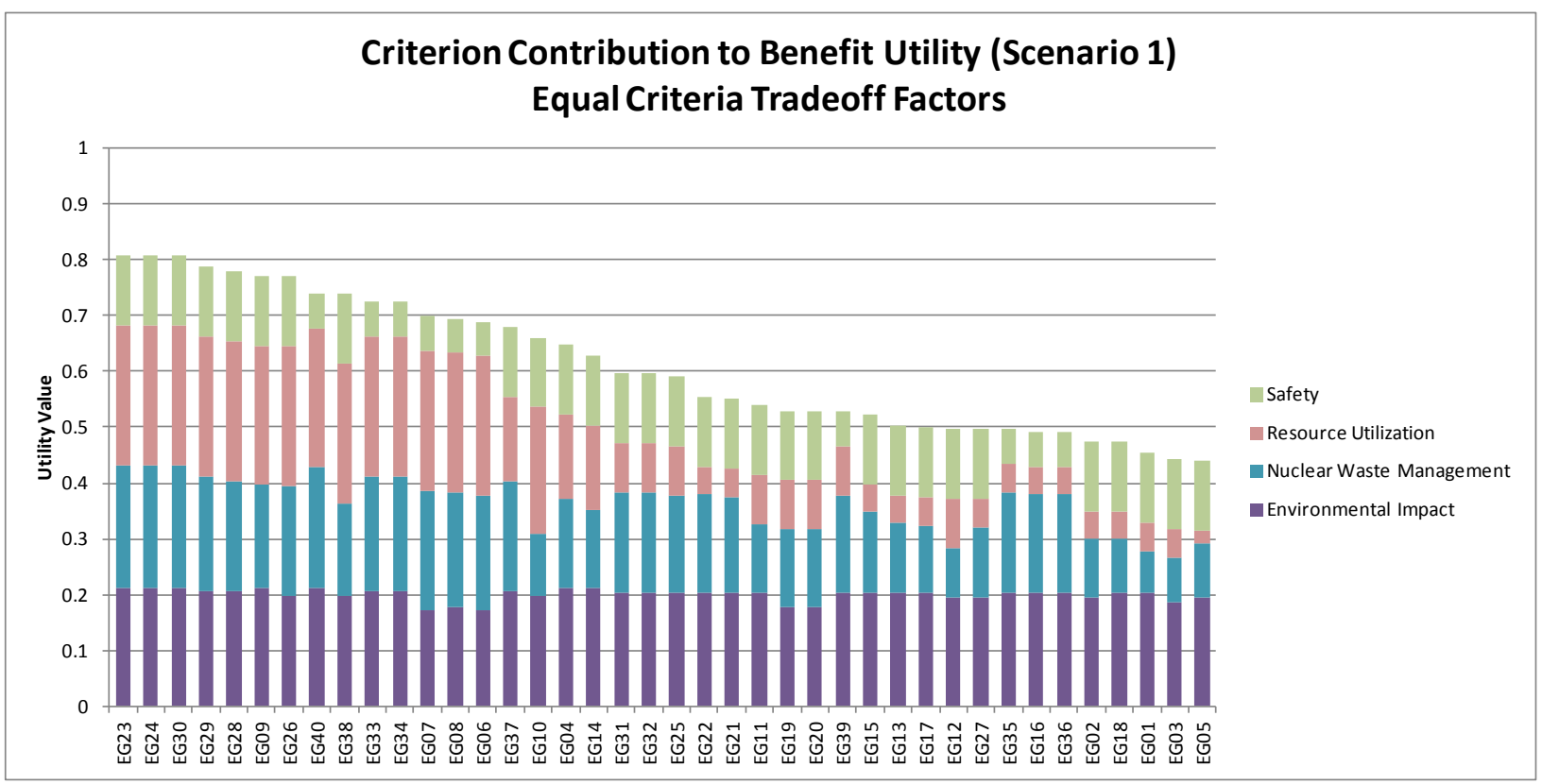

Figure 8. Criterion Contribution to Benefit Utility Report Example.

\subsubsection{Metric Contribution to Benefit Utility}

The fourth and final report in the list is the Metric Contribution to Benefit Utility report. This report is similar to the previous report except this one is by individual metric. This report generates a new spreadsheet with one summary column graph of each of the options sorted in order of decreasing benefit utility for each scenario. Within each column, the color shows the contribution of each of the metrics. This report generates a worksheet and graph for each of the scenarios selected in step 2. View/Define Scenarios. 


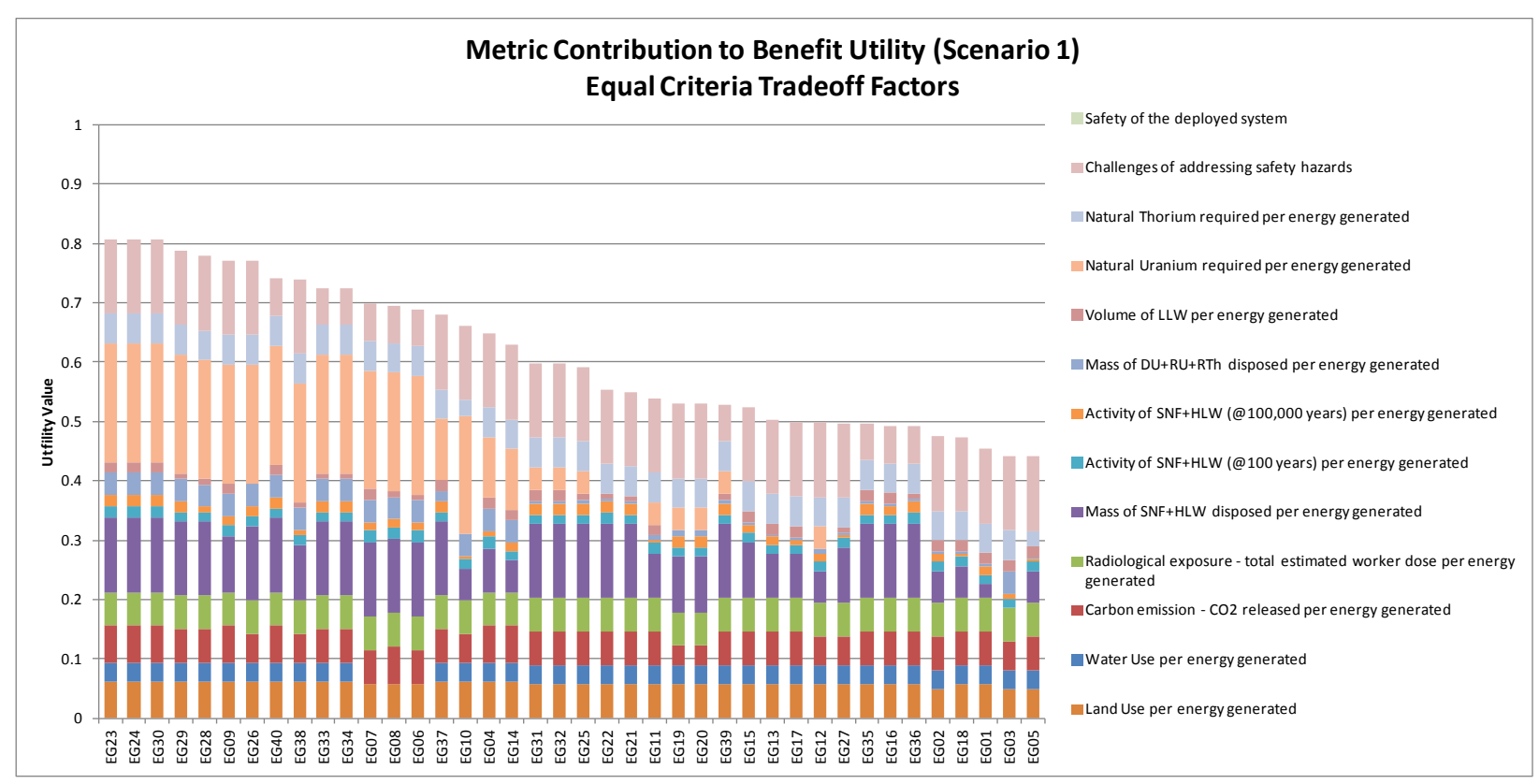

Figure 9. Metric Contribution to Benefit Utility Report Example.

\section{Performing Specific Tasks Using the SET Tool}

This section of the document outlines the steps to take to perform certain specific tasks in the SET tool.

\subsection{Determining Which Evaluation Group Best Represents My Fuel Cycle}

This feature of SET is provided to guide the user through a series of questions to determine which Evaluation Group would best represent the performance of any particular fuel cycle.

1. Click on the Find which Evaluation Group my fuel cycle is in button on the main page.

2. Step through the questions selecting answers that apply to your fuel cycle. There is a help button (question mark button) on each page to help the user with questions. As you answer questions the list of EGs on the right will update showing you which ones are still included based on your responses so far. The red EGs are once through options. The green EGs are limited recycle options. The blue EGs are continuous recycle options. 


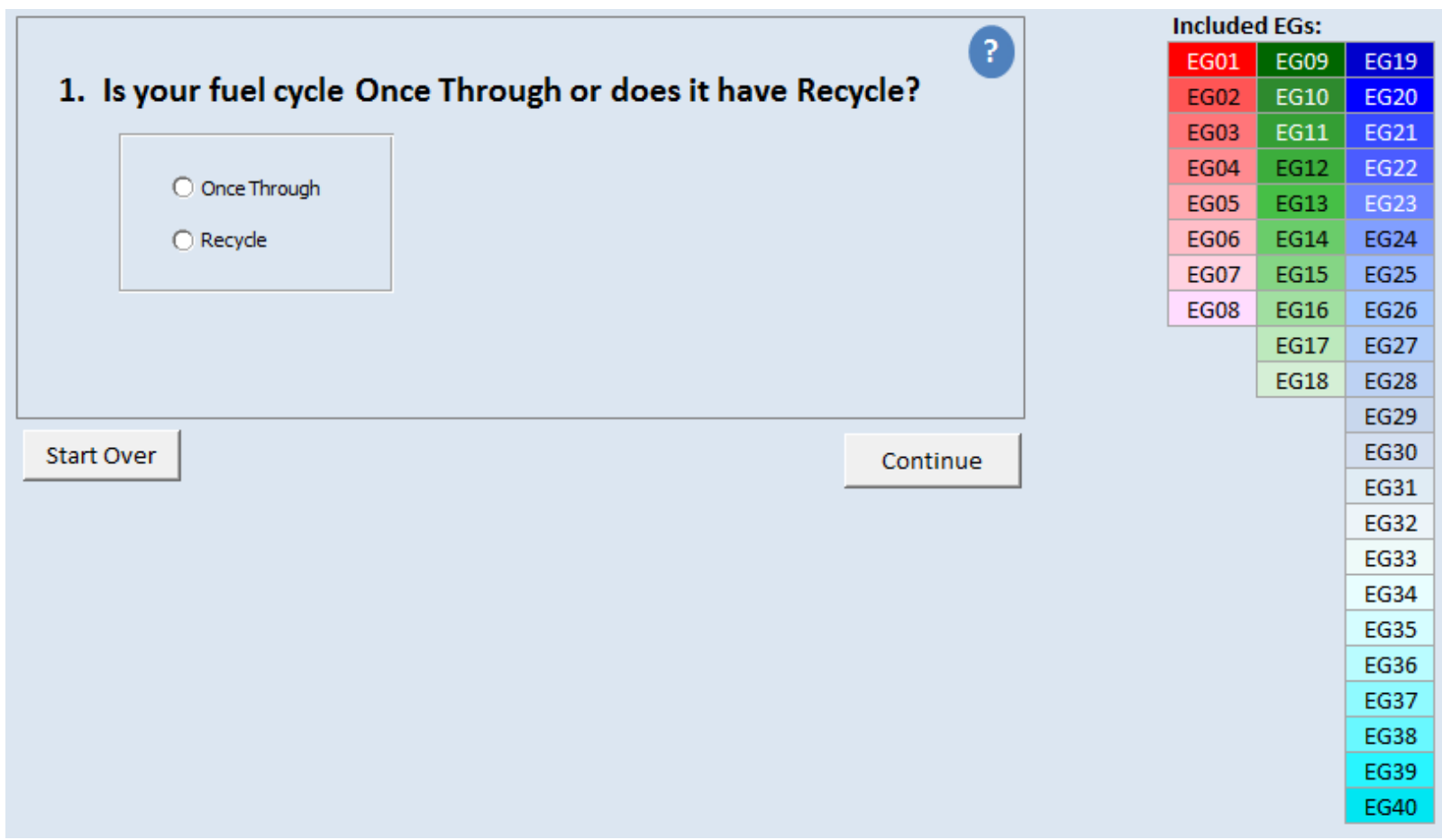

Figure 10. First Step in "Find Which Evaluation Group My Fuel Cycle Is In" Process.

3. When you get to the end the final screen will show you the Evaluation Group that best represents your fuel cycle. If you were not specific enough in your choices, SET may let you know that your fuel cycle fits into more than one Evaluation Group. If this is the case then examine the various Evaluation Groups and look at the characteristics of each of the Evaluation Groups and determine which one may best match the performance of your fuel cycle (See Figure 11). This screen also shows you a summary of each of the metric values for the selected Evaluation Group (highlighted in yellow). 


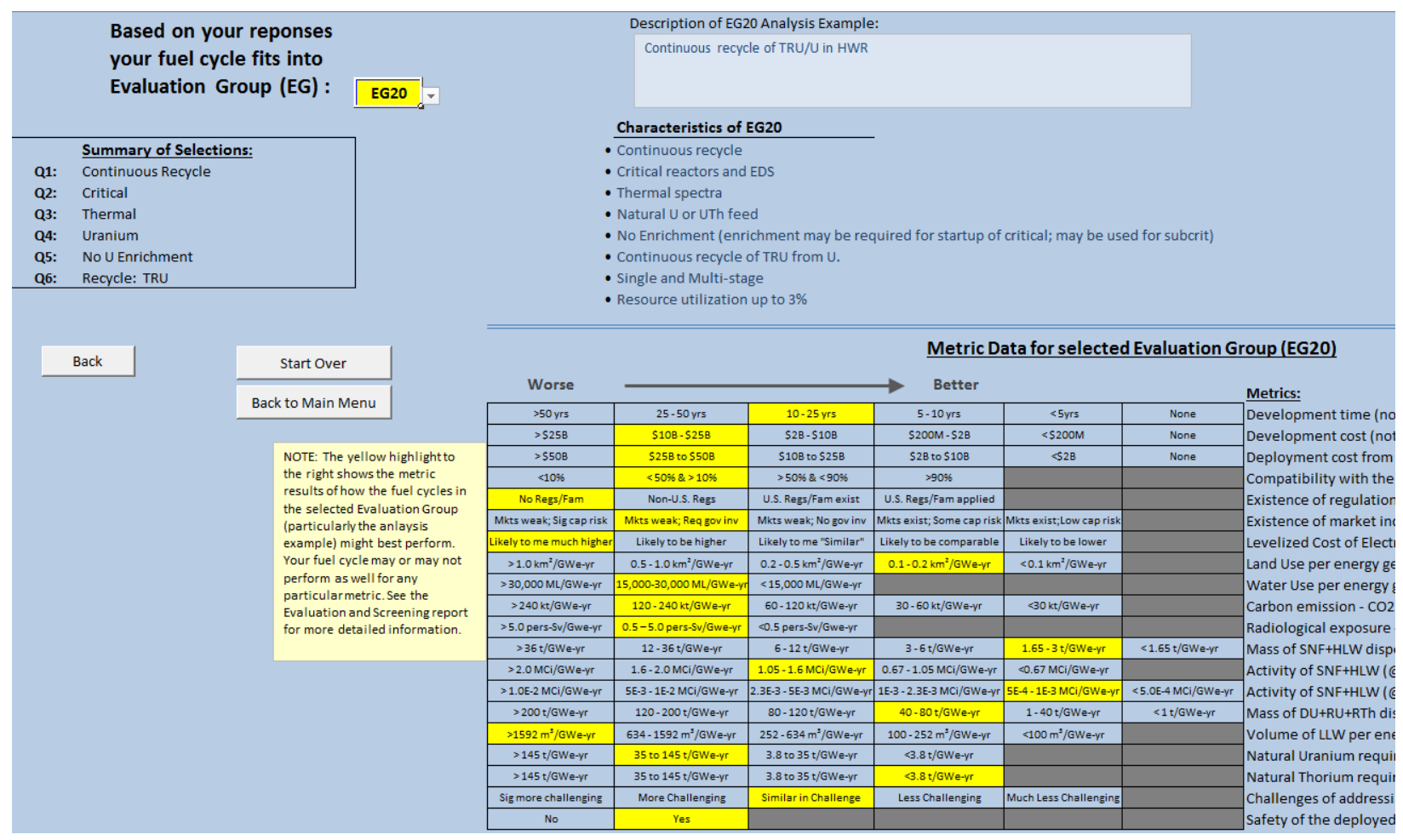

Figure 11. Final Screen for Identifying Which Evaluation Group Your Fuel Cycle Is In.

\subsection{Adding a New Option}

1. Click on the 1. View/Define Options button.

2. Scroll down to the bottom of the list and enter a name (e.g. EG41) in the Evaluation Group column and optionally enter descriptions/notes in the other columns. (Then click on the Back button to go back to the main screen).

3. Click on 3. Option Data and Assessments and using the green navigation buttons in the upper left part of the screen, navigate to your new option.

4. Next you will need to step through each of the metrics (using the blue navigation buttons in the upper left part of the screen) and enter a value at the bottom of the screen for each metric. This is done simply by pressing the Set Bin button corresponding to the bin the new option falls into. For example, if the new option needed $<5$ yrs of development for the first metric (Development Time) then you would press the set bin button just below the bin labeled " $<5$ yrs" and it will be saved. Now you can step to the next metric. (You will not need to enter a bin value for metric index 3, Development time and cost, because it is the combination of the first two metrics.) 


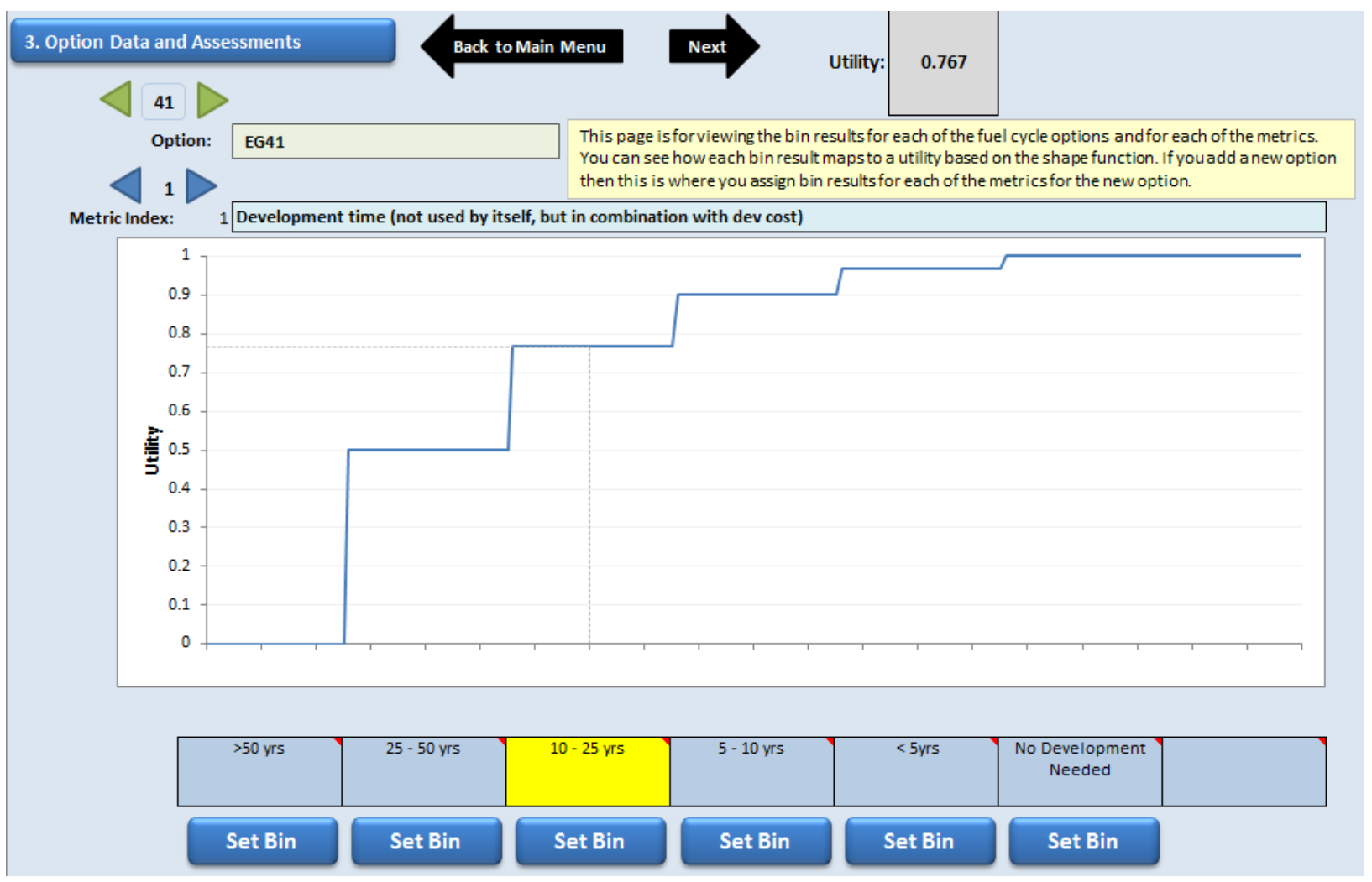

Figure 12. Adding Metric Values for a New Option.

5. When done setting a bin value for all metrics, press the Back to Main Menu button to return to the main screen or press Next. If you went back to the main screen, then press the 4. Calculate Results button to update the results to include the new option.

6. You may now proceed to the Analysis Reports page and run some reports to review how the new option performed.

\subsection{Adding a New Scenario}

1. Click on the 2.View/Define Scenarios button.

2. Scroll to the right until you see the green highlighted cells (starting with Scenario 12). Enter a scenario name at the top of the screen. Enter the percentages in the Criteria Trade-off Factor column for the Benefit Criteria to your desired values (they need to sum to 100\%). Enter the percentages for each of the metrics so that each group of metrics sum to $100 \%$.

3. Select the "Show in Output" check box at the top of the screen for the new scenario.

4. Press the Back to Main Menu button to return to the main screen.

5. Then press the 4. Calculate Results button to update the results.

6. You may now proceed to the Analysis Reports page and run a report (e.g. Benefit vs. Challenge report) to review how the new scenario affected the outcome. 